\title{
Utilização de Experimentação Remota Móvel no Ensino Médio
}

\author{
José Pedro Schardosim Simão \\ UFSC - Araranguá, SC - Brasil, E-mail: jpsimao@me.com \\ João Paulo Cardoso de Lima \\ UFSC - Araranguá, SC - Brasil, E-mail: joao.pcl@grad.ufsc.br \\ Willian Rochadel \\ UFSC - Araranguá, SC - Brasil, E-mail: willian.rochadel@ufsc.br \\ Juarez Bento da Silva \\ UFSC - Araranguá, SC - Brasil, E-mail: juarez.silva@ieee.org
}

\begin{abstract}
Resumo
Neste trabalho é relatada a experiência de desenvolvimento e implantação de uma aplicação voltada à utilização de experimentação remota em dispositivos móveis como ferramenta de apoio ao ensino e aprendizagem. A geração que hoje frequenta a escola vive rodeada de tecnologia, porém, o paradigma tradicional de ensino por vezes se mostra inflexível e acaba deixando de lado o ensino individualizado. $\mathrm{O}$ modelo apresentado é baseado na interação entre dispositivos móveis, o ambiente virtual de aprendizagem Moodle e dispositivos físicos acessados e controlados remotamente. $O$ acionamento de dispositivo físico (experimento) é efetuado através de um comando enviado a partir de um dispositivo móvel a um sistema computacional, baseado em microcontrolador, que por sua vez retorna resultado do experimento ao usuário. Todo este processo é acompanhado on-line, via streaming de vídeo, produzido por uma câmera IP. O modelo desenvolvido está sendo aplicado, através de projeto piloto, em escola de educação básica da rede pública de ensino em turmas da $2^{\mathrm{a}}$ série do ensino médio.
\end{abstract}

\section{Using Mobile Remote Experimentation in High School}

\begin{abstract}
This paper describes the experience of developing and implementing an application directed to the use of remote experimentation in mobile devices as a tool to support teaching and learning. The generation that now attends the school have a life surrounded by technology, however, the traditional paradigm of education often proves inflexible and just letting go of individualized instruction. The model presented is based on the interaction between mobile devices, the virtual learning environment Moodle and physical devices accessed and controlled remotely. The drive of the physical device (experiment) is performed through a command sent from a mobile device to a computer system based on microcontroller, which in turn returns the result of the experiment to the user. This whole process is monitored online via streaming video, produced by an IP cam. The model is being implemented through a pilot project in a public school of basic education, in the 2nd year of high school.
\end{abstract}




\section{Introdução}

O ensino e aprendizagem formais já não estão mais diretamente ligados à atividades dentro da sala de aula (Silva, 2008), e cada vez se faz mais necessária a exploração de novas práticas de ensino. A tecnologia exerce, neste sentido, função primordial de mediar e facilitar a aproximação do aluno com as disciplinas.

O atual paradigma de utilização de tecnologias na educação muito tem se limitado à sua utilização como repositório de conteúdo ou ainda do laboratório de informática tendo como objetivo a realização de pesquisas na internet. Esta prática foi por muito tempo um grande avanço, porém hoje pode ser considerada obsoleta e tem acarretado na subutilização dos recursos disponíveis nas instituições de ensino.

Os Parâmetros Curriculares Nacionais do Terceiro e Quarto Ciclo do Ensino Fundamental (1998) ressaltam a importância da utilização das tecnologias, ao definir como um dos objetivos do ensino fundamental "saber utilizar diferentes fontes de informação e recursos tecnológicos para adquirir e construir conhecimentos". Já os Parâmetros Curriculares Nacionais para o Ensino Médio (2000) definem como uma das competências que devem ser desenvolvidas pelos alunos do ensino médio "identificar variáveis relevantes e selecionar os procedimentos necessários para a produção, análise e interpretação de resultados de processos e experimentos científicos e tecnológicos", ressaltando a importância das atividades práticas.

Deve-se levar em consideração que as Novas Tecnologias da Informação e Comunicação (NTIC) já fazem parte do cotidiano da geração que hoje frequenta a escola. Os nativos digitais segundo Prensky (2001) são fluentes na linguagem digital e jogos de computador, email, Internet e telefones celulares são partes efetivas de suas vidas.

A comunicação entre os nativos digitais se dá em grande parte via mensagens de texto, SMS ou outros aplicativos de mensagens instantâneas como WhatsApp, por exemplo. A informação por esses meios é muito dispersa, e o retorno aos estímulos é muito rápido.

Assim, o ensino tradicional, muitas vezes inflexível, acaba por não valorizar as diferenças individuais dos alunos, suas diferentes formas de assimilar o conhecimento e seus diferentes ritmos de estudo.

Uma série de aspectos são fundamentais para que uma tecnologia possa ser utilizada da maneira mais eficaz e atrativa possível ao usuário:

- Usabilidade: definido por Araujo (2012 apud Rochadel, Aquino e Silva, 2012) como a capacidade de um determinado produto ser utilizado pelo indivíduo para atingir objetivos específicos em um contexto exclusivo de interação;

- Portabilidade: capacidade de ser facilmente transportado;

- Funcionalidade: cumpre com eficiência seus fins utilitários;

- Acessibilidade: possibilidade e condição de alcance para utilização por distintas parcelas da população;

- Ubiquidade: diversos dispositivos cooperando entre si para fornecer ao usuário acesso instantâneo a novos serviços de forma transparente (Cirilo, 2012).

Assim, ao tratar-se de novas formas de utilização das tecnologias, deve-se considerar a capacidade desta tecnologia de atrair o aluno e aproximá-lo dos conteúdos abordados. Por si só já são bastante atrativos os simuladores e laboratórios virtuais com experiências simuladas de 
resultados gravados; mas, além destes recursos, a utilização de recursos remotos tende a permitir uma experiência ainda mais real (Paladini, 2008).

A Experimentação Remota (RE) é uma tecnologia que permite que o usuário, mesmo à distância, controle um experimento físico, observando os resultados via streaming de vídeo. Silva (2012) observa que nestes laboratórios "os elementos são reais, seu acesso é virtual e as suas experiências reais". Diferente da simulação e dos laboratórios virtuais, os laboratórios remotos utilizam experimentos reais, o que aproxima os resultados dos obtidos pelos laboratórios presenciais (hands-on).

A utilização de experimentos proporciona um barateamento dos recursos tecnológicos, já que o mesmo experimento pode ser acessado por diversas escolas, em diversas localidades. Além disso, as configurações de equipamento necessárias para o acesso aos experimentos são mínimas, sendo o mais importante o acesso à internet.

\section{Trabalhos Correlatos}

Existe uma série de trabalhos abordando o desenvolvimento de ferramentas e a utilização de experimentação remota móvel como suporte ao ensino e aprendizagem. Rochadel, Aquino e Silva (2012) abordam o desenvolvimento de uma ferramenta para interação com experimentos remotos via dispositivos móveis.

Rochadel et. al. (2013) trata da utilização de experimentação remota em dispositivos móveis no ensino básico. Silva et. al. (2013) também discorre sobre o uso de dispositivos móveis para acesso a experimentos remotos na educação básica.

Costa (2005) já trata da utilização desta tecnologia no ensino à distância. Já GarcíaZubia e Dziabenko (2011) estudam como unir jogos e experimentação remota, explorando metodologias para o seu desenvolvimento.

\section{Dispositivos Móveis e Educação}

Com os avanços tecnológicos, os computadores de mesa passaram a dar lugar aos dispositivos móveis, como smartphones e tablets. Segundo a IDC (2013-A e 2013-B) em 2012 houve um aumento de 78\% nas vendas de smartphones, com um total de 16 milhões de unidades comercializadas. E no mercado de tablets o aumento foi ainda maior, atingindo a taxa de $171 \%$ com 3,1 milhões de tablets vendidos.

A portabilidade, facilidade de transporte destes dispositivos (Lee, Schell e Schneider, 2005 pág. 2), aliada à modernização e redução do preço de acesso às redes móveis acaba sendo um atrativo. A cobertura das redes $3 \mathrm{G}$ está presente em 3443 municípios brasileiros (Associação Brasileira de Telecomunicações, 2012), e as redes 4g já estão sendo implantadas em diversas capitais, o que torna o acesso à internet móvel mais rápido e mais fácil.

Desde grandes metrópoles como Rio de Janeiro e São Paulo até alguns municípios mais pequenos já estão oferecendo internet sem fio gratuita em locais públicos, como praças, passeios e centros comunitários. Isto aproxima a internet da grande massa, e incentiva ainda mais a utilização de dispositivos móveis.

A popularização dos dispositivos móveis permitiu o estudo de novos métodos de utilização. Com o advento do m-learning, ou mobile learning, a utilização destes dispositivos para a educação passou a ser melhor explorada, e de maneira mais eficaz. 
O m-learning pode ser definido como a utilização didática de dispositivos móveis, como smartphones e tablets. Seus princípios partem da natural evolução do ensino à distância (dlearning) e do e-learning, e mais recentemente da aprendizagem ubíqua (u-learning), processo em que aprendizagem ocorre a partir de vários lugares ao mesmo tempo e que tem tido papel fundamental no processo de modernização do ensino (Silva, 2006). Segundo a UNESCO (2012) a aprendizagem móvel requer reconceituar as potencialidades das TIC na educação, bem como seus modelos para implementação e uso.

\section{Experimentação Remota Móvel}

É possível explorar ainda mais os conceitos de experimentação remota e m-learning ao associá-los, somando os objetivos da experimentação remota aos recursos de mobilidade que estes tem a oferecer. Assim, a utilização de laboratórios remotos em aparelhos móveis tornam-se parte de alternativas e métodos para atrair a atenção dos alunos e complementar aulas teóricas em disciplinas por muitas vezes julgadas difíceis e monótonas.

A expansão dos importantes recursos da experimentação remota nos dispositivos móveis propicia a imersão do usuário em um laboratório a qualquer momento e em qualquer lugar e utilizando o seu próprio smartphone, deste modo, um novo espaço de interação que alia justamente a ideia de ambientes ubíquos.

Costa e Alves (2006) afirmam que a experimentação móvel garante o benefício concebido pela RE para a engenharia e ciências em contextos de m-learning, ou seja, a flexibilidade, a mobilidade e motivação aos alunos. Hoje em dia a qualidade de serviço de redes sem fio está em constante expansão e grande parte possuem um móvel dispositivo, o que garante a facilidade de acesso de novas aplicações desenvolvidas com base no conceito de experimentação móvel.

Nos experimentos remotos as interações são diretas com equipamentos reais. $\mathrm{O}$ usuário observa e interage determinando valores aguardando um feedback imediato das experiências realizadas. Através do streaming de vídeo, de informações e resultados obtidos é possível conferir a aplicabilidade dos conteúdos discutidos em aula.

Deste modo, permitir acesso fácil e ubíquo a um número maior de estudantes é a finalidade do RExMobile, que seu desenvolvimento é indispensável para atender à necessidade de uma aplicação móvel para interação com os experimentos remotos compatível com a grande maioria dos sistemas operacionais destes dispositivos.

\section{Arquitetura Proposta}

A discussão sobre qual abordagem para desenvolvimento móvel utilizar é recente, assim como a popularização dos dispositivos que realizam grande variedade de tarefas e o aumento do número aplicativos disponíveis. Ao iniciar um projeto, pode-se deparar com dois paradigmas de aplicações: nativas e web mobile. Estes modelos apresentam uma série de particularidades, conforme o Quadro 1, que podem motivar sua escolha. 


\begin{tabular}{|l|l|}
\hline \multicolumn{1}{|c|}{ Aplicação Nativa } & \multicolumn{1}{|c|}{ Web App } \\
\hline Instalado no dispositivo móvel & $\begin{array}{l}\text { Pode ser instalado no aparelho ou acessado via } \\
\text { navegador, mas sua base é online }\end{array}$ \\
\hline $\begin{array}{l}\text { Utiliza linguagens de programação como } \\
\text { Android, Java, Visual Basic e C\# }\end{array}$ & $\begin{array}{l}\text { Normalmente utiliza linguagens de marcação e } \\
\text { script, como HTML, CSS, Javascript e PHP }\end{array}$ \\
\hline $\begin{array}{l}\text { Para a atualização, uma nova versão deve ser } \\
\text { instalada no dispositivo }\end{array}$ & $\begin{array}{l}\text { atualizações podem ser feitas diretamente no } \\
\text { servidor }\end{array}$ \\
\hline
\end{tabular}

Quadro 1. Aplicações Nativas versus Web Apps

A seleção do modelo deve ser baseada nas necessidades específicas do projeto. Neste caso, uma aplicação móvel de experimentação remota deve necessariamente ser capaz de oferece as mesmas experiências de interação já discutidas, ou seja, ativar as experiências disponibilizadas remotamente através da com uma conexão com a internet. Este interfaceamento em desktops é feito através de páginas PHP, possibilitando a comunicação com os experimentos que estão ligados aos microservidores Web. Já na versão móvel, são utilizadas as linguagens HTML5, CSS3 e o framework jQuery Mobile para interface de usuário.

O padrão de interface adotado foi o do Windows Phone, onde a estrutura é dividida em tiles (blocos) alinhados em um grid, conforme Figura 1. Neste modelo a interface é minimalista, as cores e formas utilizadas são simples e de fácil entendimento. Cada experimento tem uma cor, que é utilizada como tema nas telas seguintes.

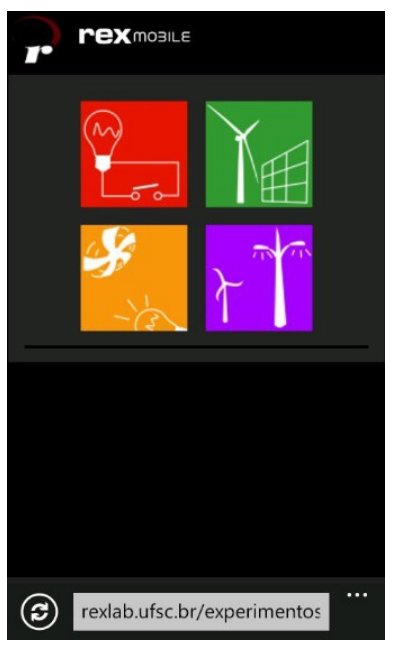

Figura . Tela inicial do aplicativo

$\mathrm{Na}$ tela inicial, o usuário tem acesso aos experimentos remotos disponíveis. Ao selecionar o experimento, o usuário tem algumas informações relevantes sobre o mesmo. 


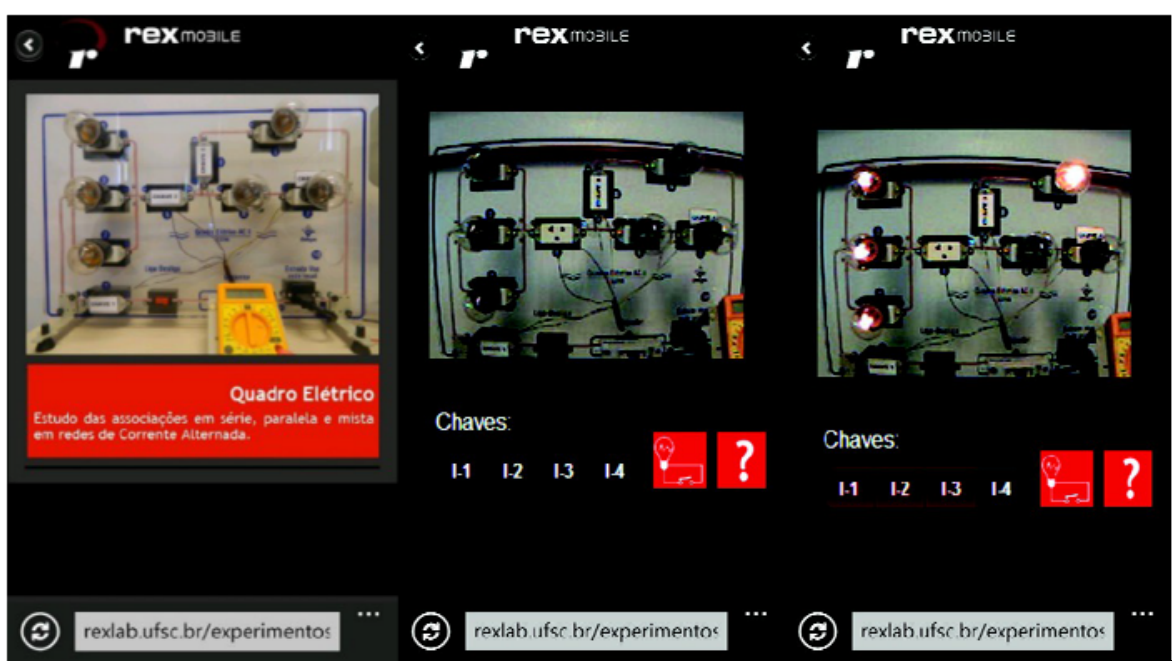

Figura . Telas de acesso ao experimento

$\mathrm{Na}$ tela seguinte, o usuário pode acionar o experimento, além de ter opções de ajuda e informações sobre o conteúdo abordado. Para garantir o fluxo entre as telas, foi adicionado um botão "Voltar" no canto superior esquerdo.

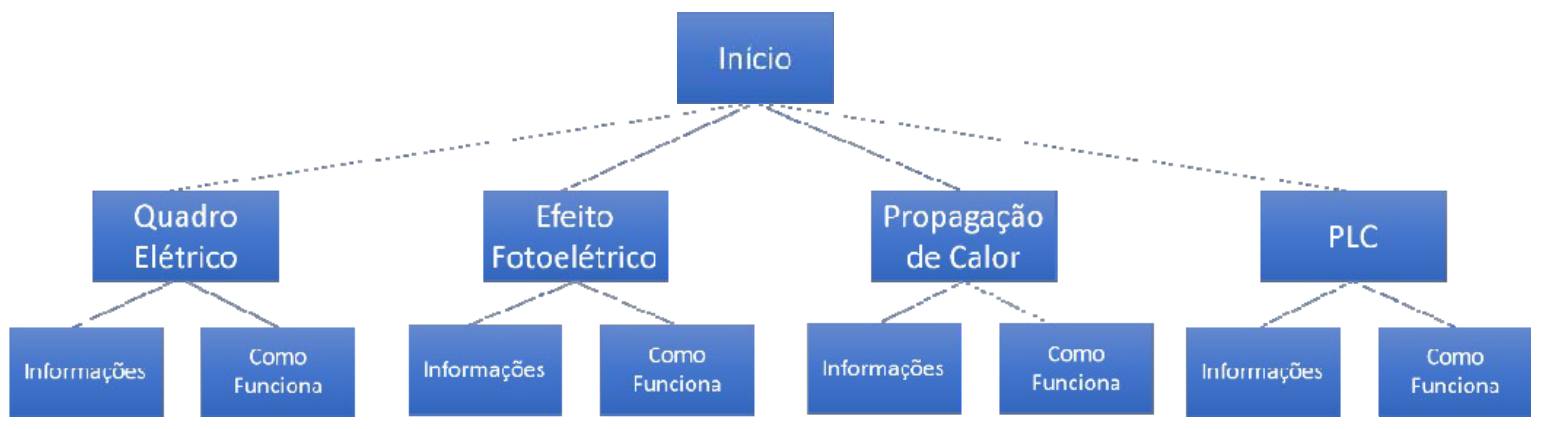

Figura . Diagrama de Navegação da aplicação

Os experimentos reais são desenvolvidos em uma arquitetura baseada em software e hardware livre, que permitem o controle através de experimentos reais através de comandos de socket enviados do dispositivo móvel via internet para uma placa microcontroladora com porta ethernet. Estes comandos servem para o controle de relés, chaves ou outros equipamentos eletrônicos. 


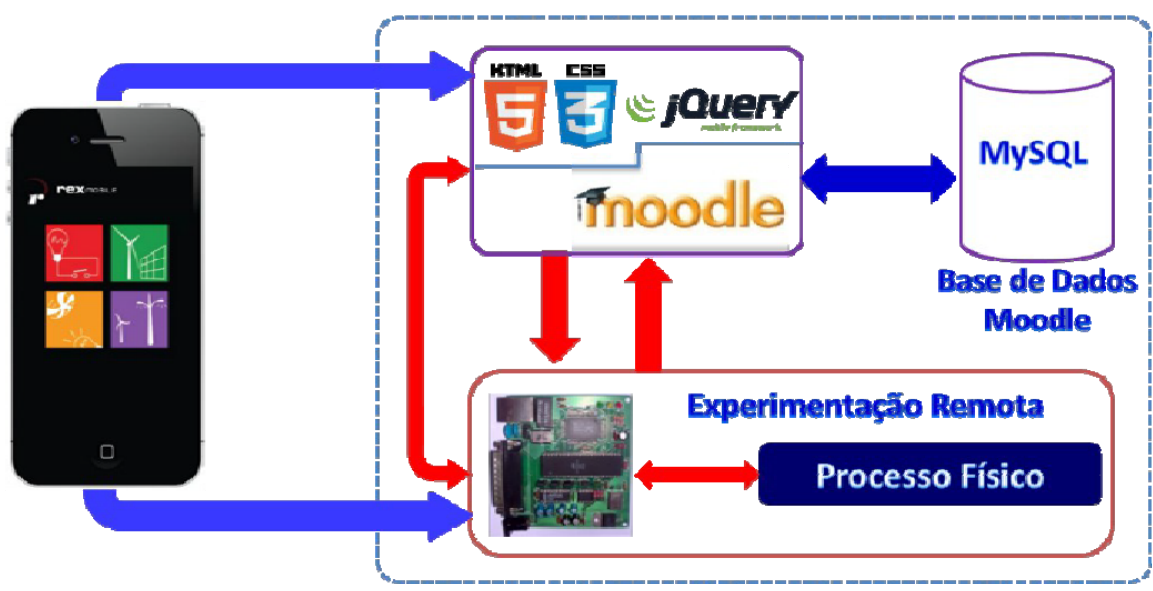

Figura . Arquitetura proposta

Em seguida, as informações recebidas pelos sensores, medidores e equipamentos são transmitidos na ordem inversa, e os sinais recebidos pelo microcontrolador são convertidos e devolvidos à interface do usuário, conforme a Figura 5. A aplicação mostra os resultados em tempo real, e imagens do experimento através de uma câmera IP.

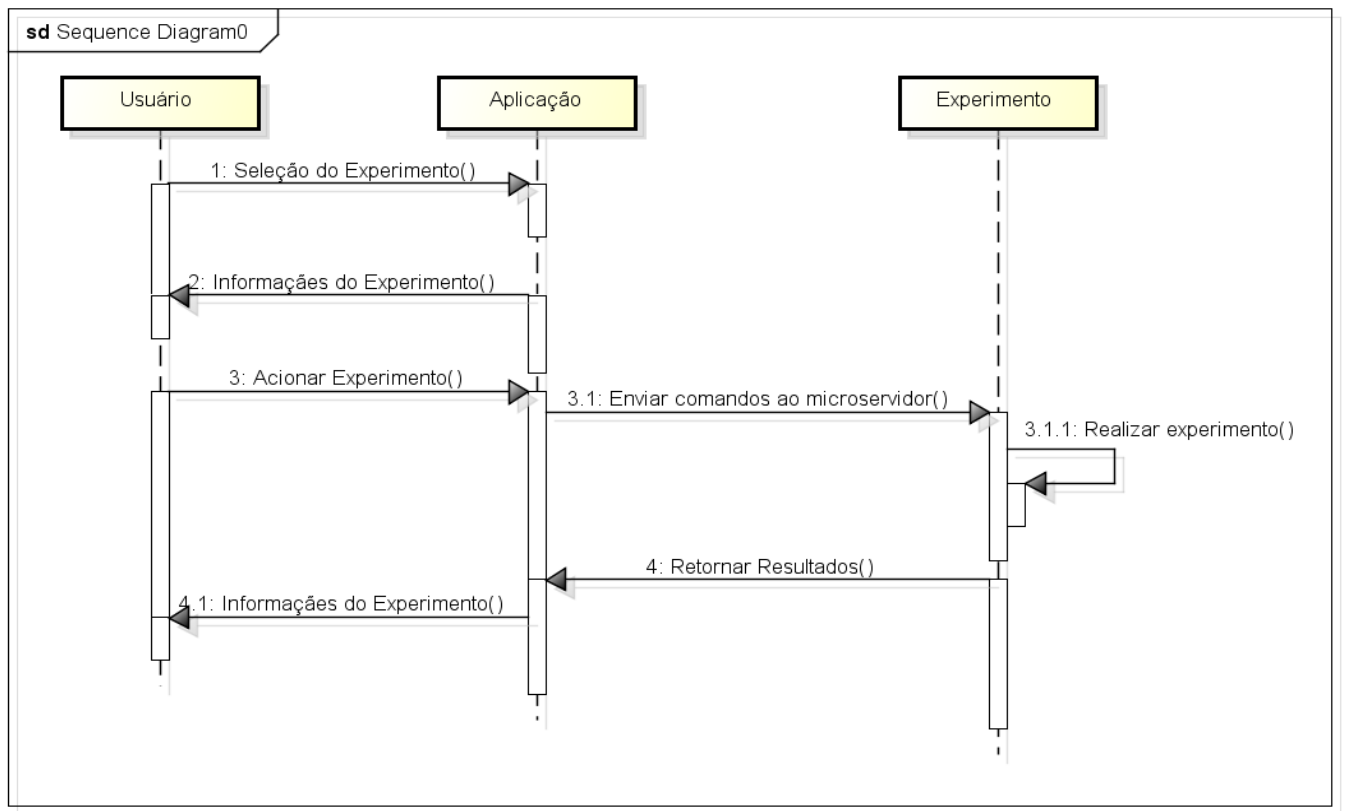

Figura . Diagrama de Sequência

Devido à incompatibilidades com os formatos e plug-ins da câmera nos dispositivos móveis, foi desenvolvido um script na linguagem JavaScript que captura um frame da câmera a cada 200 milissegundos, do qual resulta um streaming de vídeo.

O estudo do experimento é complementado através da colaboração no ambiente virtual de ensino e aprendizagem Moodle, disponibilizando material didático sobre os conteúdos abordados. Este material procura contextualizar a explicação das práticas experimentais nos eventos do cotidiano do aluno.

Ao implementar esta arquitetura para interação nos dispositivos móveis, é preciso que haja uma comunicação adequada entre os elementos e que a resposta seja o mais rápida possível. $\mathrm{O}$ 
aplicativo está em fase de testes e pode ser acessado pelo endereço "http://rexlab.ufsc.br/experimentos/mobile" ou pelo QR Code abaixo (Fig. 6).

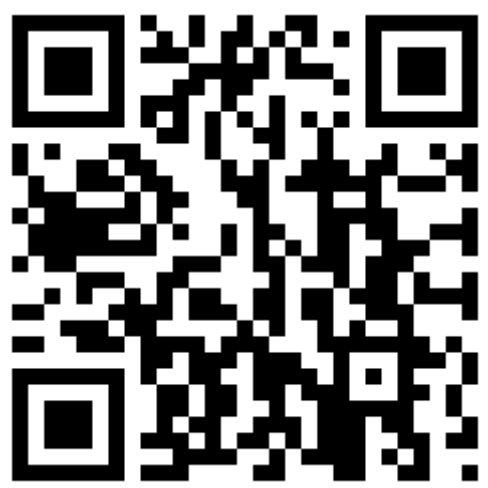

Figura : QR Code para acesso ao aplicativo

\section{Utilização no Ensino Médio}

A arquitetura discutida está sendo aplicada em fase experimental em uma escola pública, de ensino médio, em turmas da $2^{\mathrm{a}}$ série do ensino médio. A abordagem utilizada busca complementar a didática do professor com a observação experimental.

Neste contexto, o Moodle tem um papel muito importante atuando como repositório das apresentações e resumos comuns do professor de sala de aula, além da elaboração (ou ainda adaptação) de tarefas sobre o conteúdo abordado pelo experimento, agora adaptadas ao ambiente virtual. Estas tarefas incrementam as pontuações em avaliações e integram a nota trimestral, afim de aumentar ainda mais o interesse dos alunos.

A utilização da experimentação remota móvel em sala de aula pode se dar de duas maneiras: em complemento à experimentos reais, afim de proporcionar aos alunos a chance de fazer um paralelo entre diferentes tipos de aplicações da teoria, ou como única forma de experimento, o que possibilita que escolas sem recursos de laboratório possam realizar este tipo de atividades.

Outra importante forma de aplicação desta tecnologia é como lição de casa. A revisão dos conteúdos pelo aluno utilizando experimentação remota móvel e a aplicação de questionários no Moodle pode vir a auxiliar na fixação deste conteúdo na memória de longo prazo.

Algumas dificuldades e barreiras estão sendo encontradas na aplicação desta tecnologia na escola pública em questão. Alguns alunos não possuem conexão com a internet em casa, porém estes acessaram as atividades a partir da casa de colegas. Já quem procurou a escola para utilizar a internet encontrou uma série de problemas, como velocidade baixa e sinal de internet sem fio fraco.

Outro problema encontrado é a distribuição de atividades no calendário. Os conteúdos andam de acordo com os ritmos de cada turma, e poucas são as aulas disponíveis para apresentação dos experimentos. A política pedagógica da escola define que as avaliações devem ser aplicadas conforme um calendário, independente da aula e da disciplina. Além disso, inclui aulas exclusivas para incentivo da leitura, no qual os alunos tem de praticar a leitura. Estas particularidades acabam por atrasar o andamento das aulas, acarretando muitas vezes em curtos prazos para concluir conteúdo programático nos finais do ano letivo. 
A despeito das dificuldades encontradas, as escolas públicas estão recebendo recursos tecnológicos do Ministério da Educação (MEC). Conforme Portal Brasil (2012), o MEC disponibilizou o investimento de $\mathrm{R} \$ 150$ milhões para compra de 600 mil tablets para uso dos professores do ensino médio das escolas públicas no ano de 2012. Ainda em execução, este projeto do governo federal pretende oferecer mais instrumentos e formação aos professores e gestores das escolas públicas para o uso intensivo das NTICs no processo de ensino e aprendizagem.

\section{Conclusão}

Dentro de uma gama de incentivos relacionados à integração das tecnologias na educação, há a necessidade de fornecer qualidade e diversidade de conteúdo ao estudante de ensino médio, dada a extrema importância de instigá-los à participação em sala de aula. No que se refere ao ensino de física, sabe-se que é essencial a presença da experimentação em todo processo de desenvolvimento das competências do aluno.

A integração do m-learning à experimentação remota mostra-se muito mais do que um objeto educacional, ela permite estender as atividades de ensino e aprendizagem dentro e fora da sala de aulas e figura como recurso para apoio e enriquecimento das relações entre professor e aluno, o que leva a efeito o aumento da produtividade de ensino.

A ênfase nos aspectos positivos do uso de MRE no ensino médio, principalmente na disciplina de física, objetiva somar às aulas expositivas os conteúdos educacionais que expandem o ambiente de aprendizagem para além do ambiente escolar tradicional e dinamizem o uso de dispositivos móveis e da internet.

Enquanto todas estas atividades decorrem, o interesse pela comunicação de dados, arquitetura e processos envolvidos tendem a despertar a curiosidade do usuário em diversas áreas da engenharia e tecnologias. A ideia de aparatos físicos serem controlados a distância, providencia uma interessante ferramenta de apoio e demonstração não só dos benefícios do estudo com experimentos de física, mas também na abordagem tecnológica de utilidade dos campos interdisciplinares que são englobados em todo processo.

Assim, a observação não se limita somente a um olhar didático exclusivo da física, mas uma proximidade entre as ciências para permitir a construções destes sistemas, o que torna ainda mais atrativa o experimento remoto de controle via dispositivos móveis.

Como trabalhos futuros sugere-se: (i) Estudo das dificuldades e barreiras encontradas na utilização de experimentação remota móvel nas escolas públicas; e (ii) Estudo da efetividade da experimentação remota móvel como objeto de aprendizagem.

\section{Referências Bibliográficas}

ASSOCIAÇÃO BRASILEIRA DE TELECOMUNICAÇÕES, TIM supera Oi e retoma $3^{\mathrm{a}}$ posição no ranking de cobertura 3G. Telebrasil. Disponível em: $<$ http://www.telebrasil.org.br/sala-deimprensa/na-midia/3491-tim-supera-oi-e-retoma-3-posicao-no-ranking-de-cobertura-3g $>\quad$ Acesso em 27 Abr. 2013. 
CIRILO, E. C. Computação Ubíqua: definição, princípios e tecnologias. Disponível em: $<$ http://academia.edu/1733697/Computacao_Ubiqua_definicao_principios_e_tecnologias $>$ Acesso em 27 Abr. 2013.

COSTA, R. Tele-Experimentação Móvel (Mobile Remote Experimentation): Considerações sobre uma área emergente no ensino à distância. Disponível em: $<$ http://ave.dee.isep.ipp.pt/ rjc/Docs/2005/NewsletterISEP2005/NewsLetterISEP.pdf> Acesso em 30 Abr. 2013.

COSTA, R. J; ALVES, G. R., Mobile experimentation innovating education to the 'mobile phone' generation. Disponível em: <ave.dee.isep.ipp.pt/ rjc/Docs/2006/ECUMICT06/paperECUMICT 06.pdf>. Acesso em 27 Abr. 2013.

FILATRO, A. Design Instrucional na prática. São Paulo: Pearson Education do Brasil, 2008. 192 pg.

GARCÍA-ZUBIA, J. E DZIABENKO, O. Remote Experiments and Online Games: how to merge them? IEEE Global Engineering Education Conference (EDUCON), 2011. DOI 10.3991/ijep.v1i1.1601

IDC, Mercado brasileiro de celulares encerrou $2012 \mathrm{com}$ a marca de 59,5 milhões de unidades comercializadas. Disponível em: $<$ http://br.idclatin.com/releases/news.aspx?id=1462>Acesso em 10 maio 2013.

IDC, Mercado de tablets no Brasil foi o que mais cresceu em 2012, revela estudo da IDC. Disponível em: <http://br.idclatin.com/releases/news.aspx?id=1457> Acesso em 10 maio 2013.

LEE, V.; SCHNEIDER, H.; SCHELL R., Aplicações Móveis: Arquitetura, projeto e desenvolvimento. São Paulo: Pearson Education do Brasil, 2005. Cap. 1, p. 2.

MEC, Parâmetros Curriculares Nacionais do Terceiro e Quarto Ciclo do Ensino Fundamental: Ciências Naturais. Brasília: Ministério da Educação / Secretaria de Ensino Fundamental, 1998. 138 p.

MEC, Parâmetros Curriculares Nacionais do Ensino Médio: Ciências Naturais. Brasília: Ministério da Educação/Secretaria de Ensino Básico, 2000. 58 p.

PALADINI, S.; SILVA, J.B.; ALVES, G.R.; FISCHER, B.T.; ALVES, J.B., Using Remote Lab Networks to Provide Support to Public Secondary School Education Level. presented at 11th IEEE International Conference on CSEWORKSHOPS '08. Computational Science and Engineering Workshops. São Paulo, SP, 2008. 
PRENSKY, M. Digital natives, digital immigrants. On the Horizon, vol. 9, ed. 5, pp. 1-6. ISSN: 1074-8121. MCB University Press, 2001.

PROFESSORES DO ENSINO MÉDIO DE ESCOLAS PÚBLICAS RECEBERÃO TABLETS NO SEGUNDO SEMESTRE. Portal Brasil. Disponível em: $<$ http://www.brasil.gov.br/noticias/arquivos/2012/02/03/professores-do-ensino-medio-de-escolaspublicas-receberao-tablets-no-segundo-semestre> Acesso em 10 maio 2013.

ROCHADEL, W. et. al. Educational application of remote experimentation for mobile devices. 10th International Conference on Remote Engineering and Virtual Instrumentation (REV), 2013. DOI 10.1109/REV.2013.6502905

ROCHADEL, W.; AQUINO, E. L. C.; SILVA, J. B., Desenvolvimento de aplicação para interfaceamento com experimentos remotos por smarthphones. Revista Novas Tecnologias na Educação, vol. 10, nº 1. ISSN 1679-1916.

SILVA, B. A Tecnologia é uma Estratégia para a Renovação da Escola. Movimento: Revista da Faculdade de Educação da Universidade Federal Fluminense, no ${ }^{0}$, pp. 28-44, ISSN: 15180344, Tecnologia Comunicação e Educação. Rio de Janeiro: 2002.

SILVA, J. B.; ALVES, J. B. M.; GIRARDI, M. M. C., A utilização da experimentação remota como suporte à ambientes colaborativos de aprendizagem. International Computer Aided Blended Learning Conference, Florianópolis: 2008.

SILVA, J. B.; ROCHADEL, W.; MARCELINO, R., Utilização de NTIC's Aplicadas a Dispositivos Móveis. IEEE Rita,Vol. 7, Núm. 3, Ago. 2012. ISSN 1932-8540.

SILVA, J. B. A Utilização de Experimentação Remota como Suporte para ambientes colaborativos de aprendizagem. Florianópolis: UFSC, 2007. Tese de Mestrado.

UNESCO, Mobile Learning and Policies: Key Issues to Consider. United Nations Educational, Scientific and Cultural Organization, Paris: 2012. ISSN 2227-5029. 